\title{
Immunosuppressive therapy induced coronary vasospasm and acute myocardial infarction in a patient undergoing new renal transplantation
}

\author{
Ismail Biyik ${ }^{1}$, Ibrahim Faruk Akturk², Ahmet Arif Yalcin², Omer Celik², Ender Oner \\ ${ }^{1}$ Department of Cardiology, Usak State Hospital, Usak, Turkey \\ 2Department of Cardiology, Mehmet Akif Ersoy Thoracic and Cardiovascular Surgery Training and Research Hospital, Istanbul, Turkey \\ Postep Kardiol Inter 2015; 11, 2 (40): 141-145 \\ DOI: $10.5114 /$ pwki.2015.52288
}

\begin{abstract}
Immunosuppressant agents such as calcineurin inhibitors (CNI) used after solid organ transplantation may cause endothelial dysfunction, and coronary and renal arterial vasospasm. We report a patient presenting acute ST segment elevation myocardial infarction (STEMI) at the second week of renal transplantation. In the case of STEMI in patients with solid organ transplants under immunosuppressive therapy with CNI, coronary vasospasm associated with these drugs should be kept in mind before starting any interventional procedure. High dose nitroglycerine may immediately resolve tacrolimus or cyclosporine A induced coronary vasospasm. Calcium channel blockers should immediately be added to treatment because of the short half-life of nitroglycerine.
\end{abstract}

Key words: vasospasm, tacrolimus, mycophenolate mofetil, ST segment elevation myocardial infarction, transplantation.

\section{Introduction}

Transplantation specific risk factors added to the conventional risk factors may contribute to the development of cardiovascular disease (CVD) in transplant patients. After renal transplantation, specific factors related to transplantation may cause the development of cardiovascular events [1]. Immunosuppressant agents such as calcineurin inhibitors (CNI), especially cyclosporine $A$ (CSA) and tacrolimus, have adverse cardiovascular effects in transplant patients [1, 2]. These immunosuppressive agents may cause endothelial dysfunction, and coronary and renal arterial vasospasm [2, 3].

Here, we report a female patient presenting acute ST segment elevation myocardial infarction (STEMI) at the second week of renal transplantation. Although coronary artery vasospasm seems to be a multi-factorial disease which is generally not related to the conventional risk factors for CVD [4], in this report, we aim to draw attention to coronary vasospasm associated with immunosuppressant therapy in patients undergoing solid organ transplantation.

\section{Case report}

A 53-year-old female patient presented to the emergency department with chest pain lasting for $1 \mathrm{~h}$. Her medical history was remarkable for hypertension, diabetes mellitus and renal failure. She had undergone cadaveric renal transplantation 2 weeks before. She was under hemodialysis treatment for 9 months before renal transplantation. Prior to the renal transplantation, she had diagnostic coronary angiography revealing non-obstructive coronary atheroma. She was under immunosuppressive therapy with tacrolimus $2 \mathrm{mg} /$ day, mycophenolate mofetil (MMF) $3 \mathrm{~g} /$ day, and prednisone $10 \mathrm{mg} /$ day and receiving subcutaneous insulin. On admission, her blood pressure was 180/80 mm Hg, heart rate was $50 \mathrm{bpm}$ and physical examination showed hemodynamically Killip class 1 findings and an incision scar at the right lower quadrant; ECG showed ST segment elevations in leads DII, DIII, aVF and reciprocal findings in precordial leads (Figure $1 \mathrm{~A}$ ). 5000 units of conventional heparin were administered by the intravenous route and $600 \mathrm{mg}$ clopidogrel and $300 \mathrm{mg}$ acetylsalicylic acid were given orally.

Corresponding author:

Ismail Biyik MD, Asc. Prof., Department of Cardiology, Usak State Hospital, 64100 Usak, Turkey, phone: +90 5424173209 ,

e-mail: ismailbiyikmd@yahoo.com

Received: 31.05.2014, accepted: 9.03.2015. 


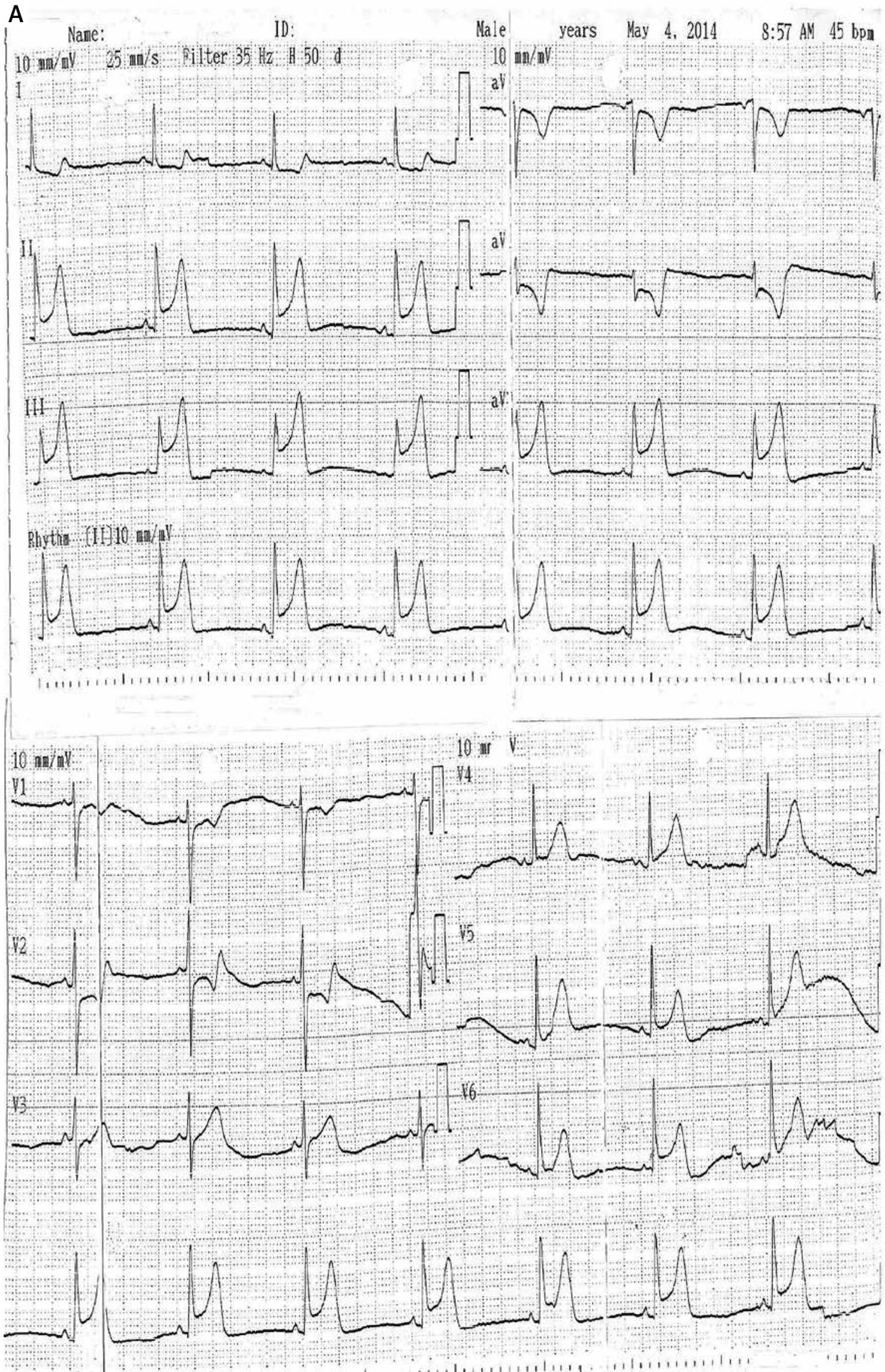

Figure 1. A - Electrocardiogram at presentation 


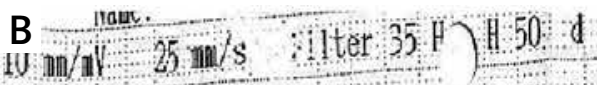

) 10 ant

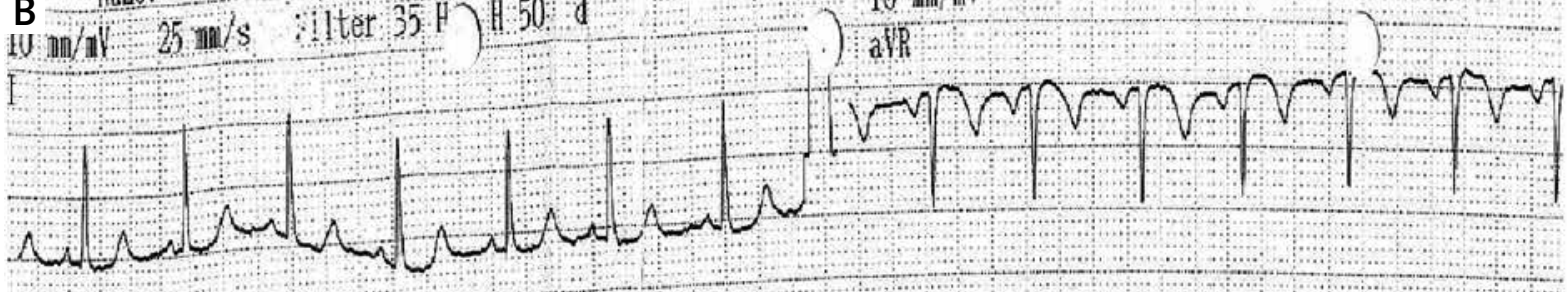

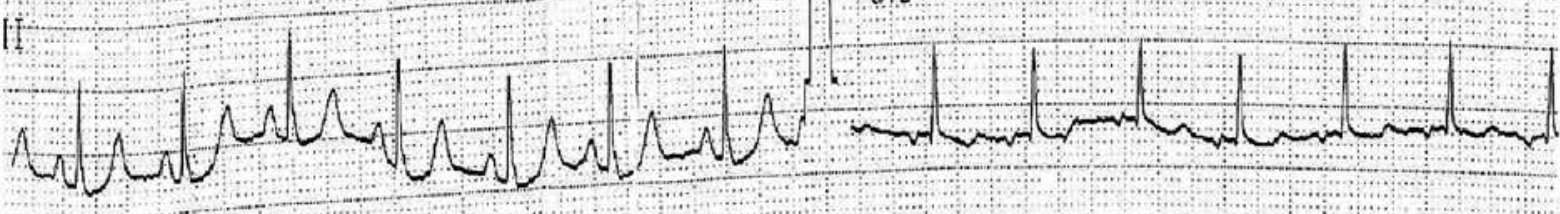

all

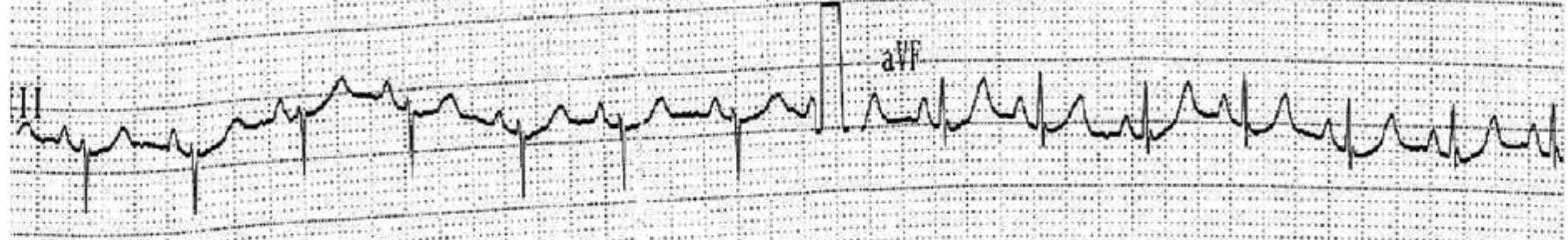

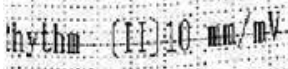

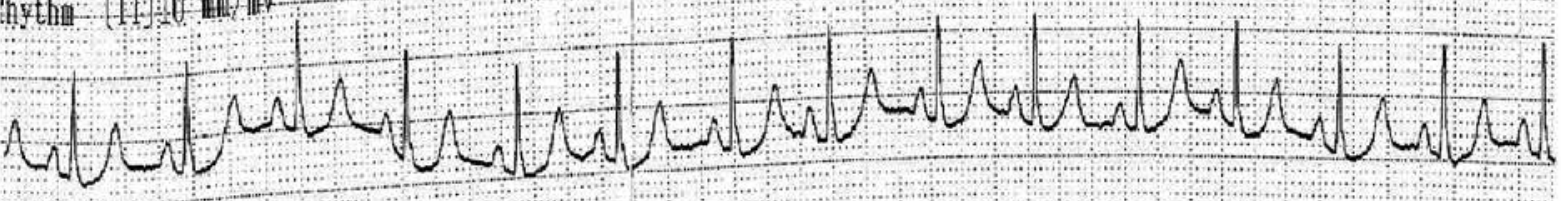

-...

…

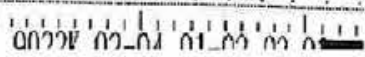

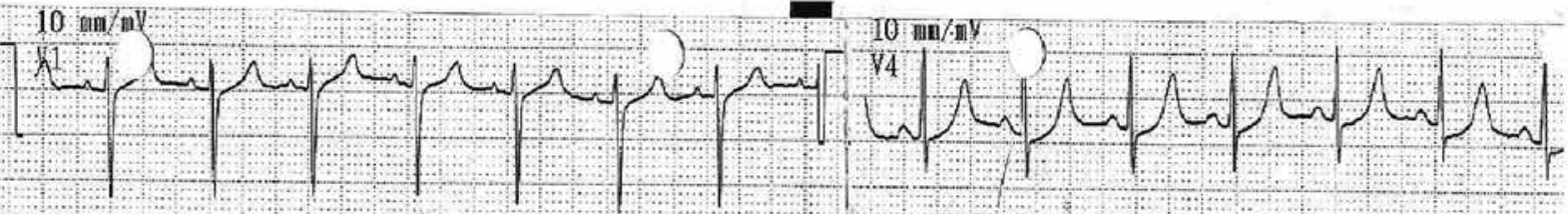

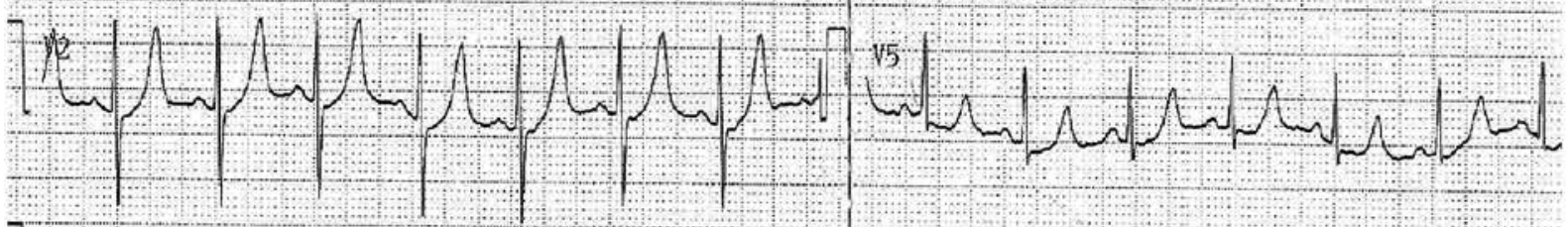

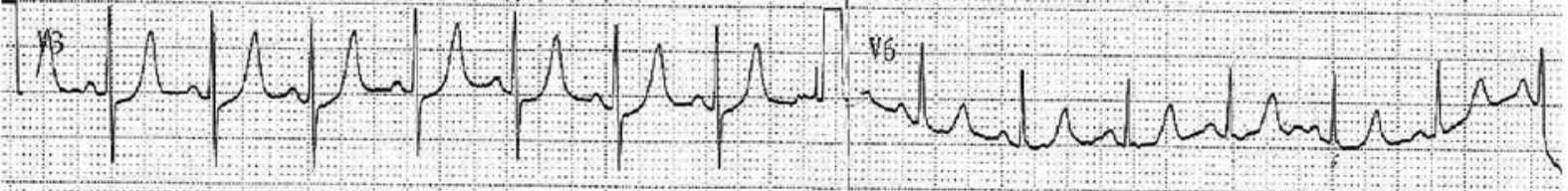

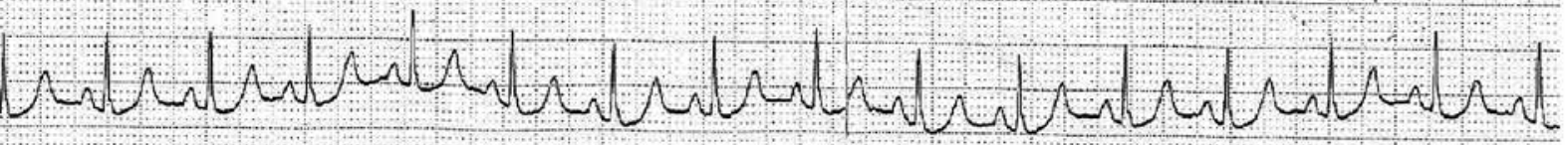

Figure 1. B - Electrocardiogram after intracoronary nitroglycerine administration 

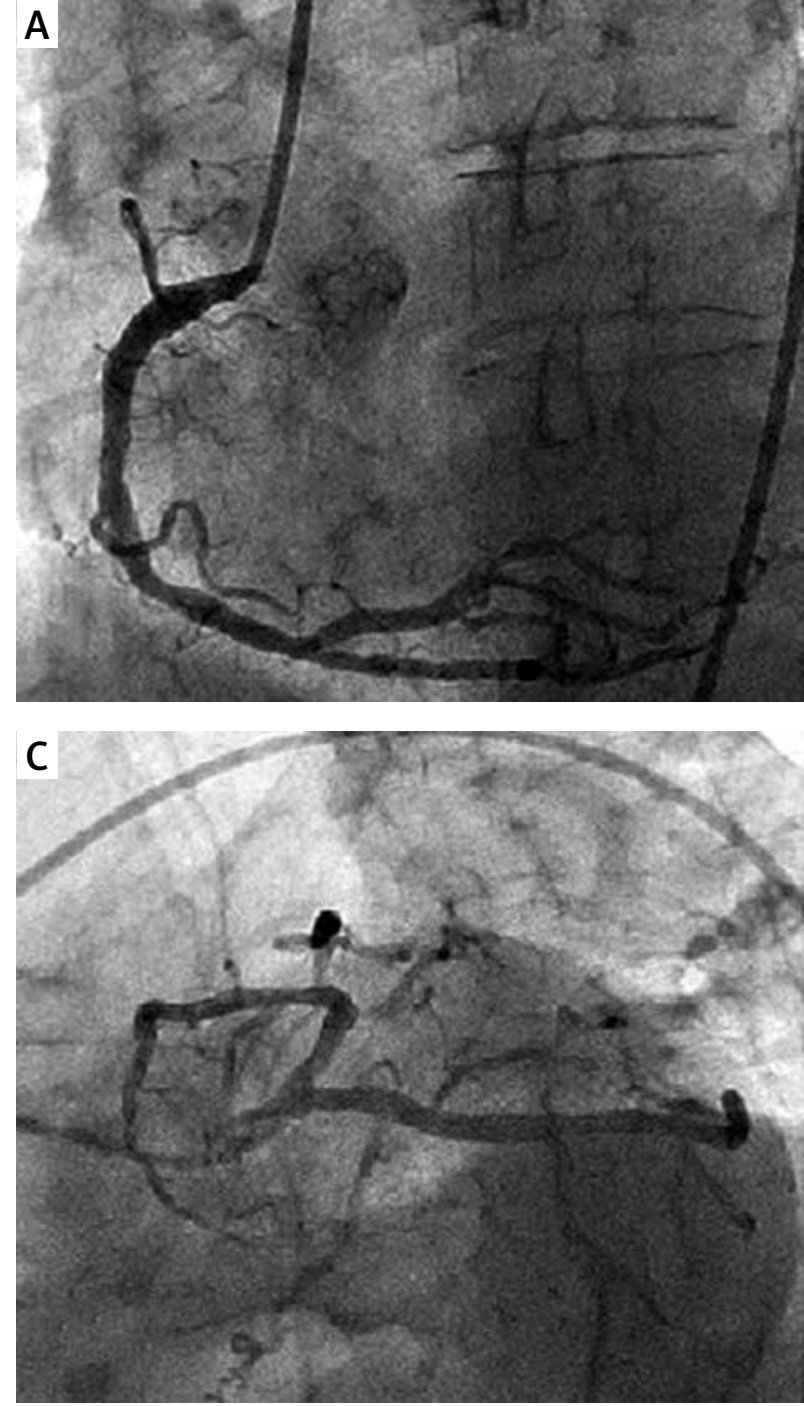

She was taken to the emergency catheterization room. Diagnostic angiography showed diffuse coronary artery disease of the right coronary artery (RCA) (Figure 2 A), approximately $90 \%$ stenosis at the ostium of the left circumflex artery (LCX) and $85 \%$ stenosis at the ostium of the left anterior descending artery (LAD) (Figure $2 \mathrm{~B}$ ). After intracoronary administration of $500 \mu \mathrm{g}$ of nitroglycerin, critical stenoses at the ostia of the LCX and LAD disappeared (Figure $2 \mathrm{C}$ ), the patient's chest pain resolved and ST segment elevations in leads DII, DIII, aVF and precordial leads and reciprocal findings on ECG returned to baseline (Figure $1 \mathrm{~B}$ ). Urgent echocardiography revealed normal ventricular systolic functions with ejection fraction > 55\% and no wall motion abnormality. In total, $75 \mathrm{ml}$ of iso-osmolar contrast agent was used during angiography. Forty mg of methyl prednisolone was given as a stress dose because the patient was on steroid therapy after renal transplantation. Hydration with $150 \mathrm{ml} / \mathrm{h}$ saline and verapamil $120 \mathrm{mg}$ twice daily were started and the patient was taken to the coronary care unit. After an

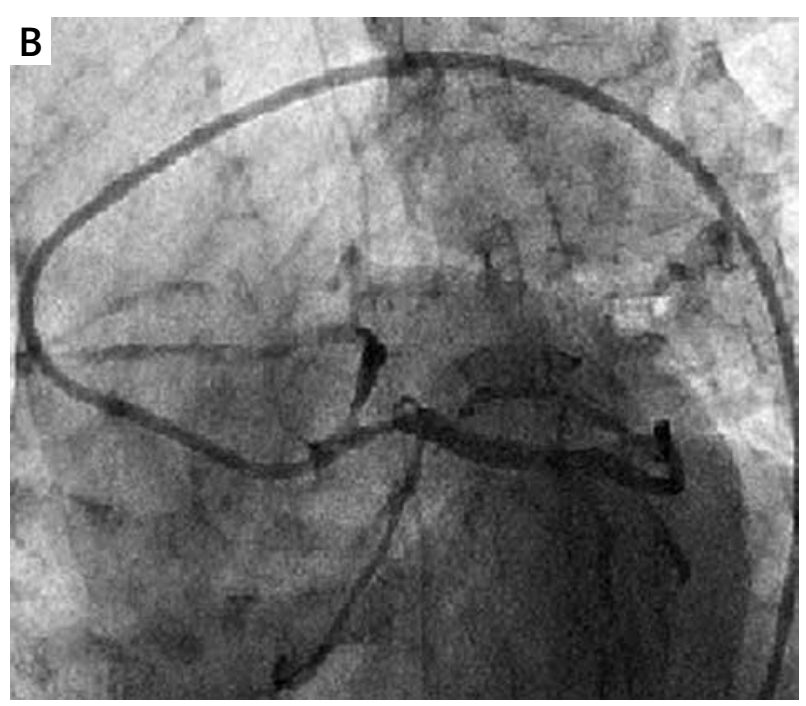

Figure 2. A - Right coronary angiography showing diffuse coronary artery disease. B - Left coronary angiography showing critical stenotic lesions at the ostia of the LAD and LCX arteries. C - Left coronary angiography demonstrating resolution of critical lesions at the ostia of the LAD and LCX arteries after intracoronary nitroglycerine administration

uneventful course she was discharged from hospital with normal cardiac and renal functions.

\section{Discussion}

Although coronary artery vasospasm seems to have different mechanisms and precipitating factors [4], calcineurin inhibitors are known to be potent vasoconstrictors and may cause coronary vasospasm as well as renal arteriolar spasm. In our case, critical stenoses at the ostia of the LCX and LAD were considered as coronary vasospasm due to tacrolimus (1) and to a lesser extent to mycophenolate mofetil (2), and high dose intracoronary nitroglycerin was given. The patient responded dramatically; chest pain and ECG findings were resolved without requiring further interventional procedures. According to our knowledge, there is no report of acute myocardial infarction associated with tacrolimus- and MMF-induced coronary vasospasm. Generalized sympathetic system excitation resulting from calcineurin inhibition with CSA and tacrolimus has been suggested 
as a responsible mechanism of their vasoconstrictor effects but not the sole mechanism [5]. Can et al. reported that tacrolimus does not alter nitric oxide production in endothelium but impairs relaxant responses, possibly through changes in receptor activation or creating an imbalance between relaxant and contracting factors within the endothelium in favor of the contracting factors in rat thoracic aortas and coronary arteries [6]. Endothelin 1 (ET-1) is a potent vasoconstrictor and increases vascular smooth muscle cell proliferation and fibrosis. It has been reported that ET-1 levels rise after isograft implantation and ET 1 may have an important role both in $\mathrm{CNI}$-induced renal vasoconstriction, sodium retention and hypertension and in endothelial vasculopathy in chronic allograft rejection [7]. It has also been reported that endothelin receptor antagonists (ERAs) can reduce $\mathrm{CNI}$-induced renal vasoconstriction, hypertension and proteinuria, and preserve renal functions in animal studies of renal transplantation [7]. The ERAs have also been shown to improve transplanted organ functions, structure and ischemic reperfusion injury in animal models of lung and liver transplantation [7]. Studies report that ambrisentan can be administered safely in conjunction with tacrolimus and mycophenolate [7]. Calcium channel blockers have been reported to be the most beneficial drugs for ameliorating CNI-induced vasoconstriction, and their use may improve renal functions after kidney transplantation [8]. It has also been reported that theophylline, a nonselective adenosine receptor antagonist and phosphodiesterase inhibitor, may reverse vasoconstriction induced by tacrolimus and may improve renal functions [9].

\section{Conclusions}

In cases of acute coronary syndrome in patients with kidney or other solid organ transplants under immunosuppressive therapy with $\mathrm{CNI}$ and MMF, coronary vasospasm associated with immunosuppressive drugs should be kept in mind before starting any interventional procedure. High-dose nitroglycerine may immediately resolve CNI-induced coronary vasospasm. Calcium channel blockers should be added to this treatment as soon as possible because of the short half-life of nitroglycerine.

\section{Conflict of interest}

The authors declare no conflict of interest.

\section{References}

1. Seino Y, Hori M, Sonoda T. Multicenter prospective investigation on cardiovascular adverse effects of tacrolimus in kidney transplantations. Cardiovasc Drugs Ther 2003; 17: 141-9.

2. Jeanmart H, Malo O, Carrier M, et al. Comparative study of cyclosporine and tacrolimus vs newer immunosuppressants mycophenolate mofetil and rapamycin on coronary endothelial function. J Heart Lung Transplant 2002; 21: 990-8.
3. Textor SC, Wiesner R, Wilson DJ, et al. Systemic and renal hemodynamic differences between FK506 and cyclosporine in liver transplant recipients. Transplantation 1993; 55: 1332-9.

4. Hung MJ, Hu P, Hung MY. Coronary artery spasm: review and update. Int J Med Sci 2014; 11: 1161-71.

5. Gardiner SM, March JE, Kemp PA, et al. Regional haemodynamic effects of cyclosporine $A$, tacrolimus and sirolimus in conscious rats. Br J Pharmacol 2004; 141: 634-43.

6. Can C, Erol A, Cinar M, et al. Therapeutic concentrations of tacrolimus do not interfere with endothelial nitric oxide synthesis in rat thoracic aortas and coronary arteries. J Cardiovasc Pharmacol 2007; 50: 399-405.

7. Raina A, Horn ET, Benza RL. The pathophysiology of endothelin in complications after solid organ transplantation: a potential novel therapeutic role for endothelin receptor antagonists. Transplantation 2012; 94: 885-93.

8. Mangray M, Vella JP. Hypertension after kidney transplant. Am J Kidney Dis 2011; 57: 331-41.

9. McLaughlin GE, Schober M, Perez M, et al. Benefit of theophylline administration in tacrolimus-induced nephrotoxicity in rats. Pediatr Nephrol 2003; 18: 860-4. 